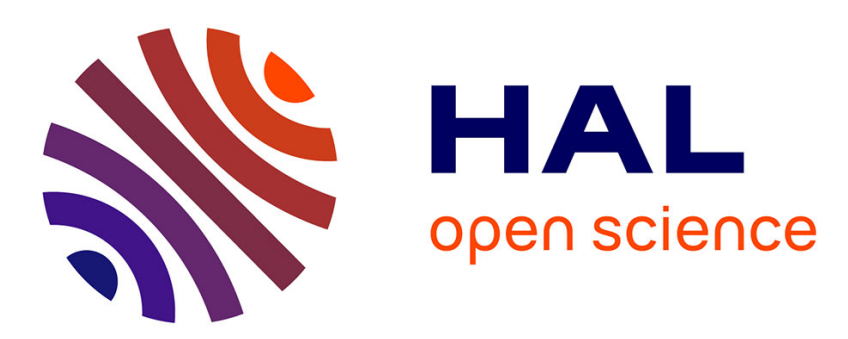

\title{
Structured Control for a Satellites Platoon Formation in Low Earth Orbit Using Youla-Parametrization.
}

\author{
F. Demourant, J.P. Chrétien
}

\section{To cite this version:}

F. Demourant, J.P. Chrétien. Structured Control for a Satellites Platoon Formation in Low Earth Orbit Using Youla-Parametrization.. The European Control Conference 2009, Aug 2009, BUDAPEST, Hungary. hal-01060311

\section{HAL Id: hal-01060311 \\ https://hal-onera.archives-ouvertes.fr/hal-01060311}

Submitted on 3 Sep 2014

HAL is a multi-disciplinary open access archive for the deposit and dissemination of scientific research documents, whether they are published or not. The documents may come from teaching and research institutions in France or abroad, or from public or private research centers.
L'archive ouverte pluridisciplinaire HAL, est destinée au dépôt et à la diffusion de documents scientifiques de niveau recherche, publiés ou non, émanant des établissements d'enseignement et de recherche français ou étrangers, des laboratoires publics ou privés. 


\title{
Structured Control for a Satellites Platoon Formation in Low Earth Orbit Using Youla-Parametrization
}

\author{
Fabrice Demourant and Jean-Pierre Chrétien
}

\begin{abstract}
The paper presents the design and analysis of a control strategy appropriate in the case of two or more spacecrafts in low Earth orbit and in close along-track configuration. The well known dynamics of the relative motion are briefly recalled and their decoupling properties allow to restrict the multivariable control design to an in-plane problem. An $H_{\infty}$ synthesis is used to obtain a control law for a formation of two satellites. The control of a formation of four satellites, in the case of local measurements between each satellite and the preceding one, falls in the framework of structured control. A Youla-parameter based technique (convex synthesis) allows to use convex optimization to improve the initial blockdiagonal structure derived from the two-satellites controllers, with a great flexibility to take into account frequency and time constraints.
\end{abstract}

\section{INTRODUCTION}

The forthcoming missions involving formation flying are mostly motivated by the need of large focal length or high angular resolution of optical telescopes. Due to the required precision of the relative motions of the vehicles in these situations, the altitude of the orbits must be sufficiently high to avoid differential atmospheric drag and gravity gradient. In the case of radar missions involving active antennas, the low earth orbit cannot be avoided and these disturbances become very important.

An example of such a mission is the ROMULUS project (whose French acronym means something like Orbital Multisatellite Radars dedicated to Monitoring): in this in-house Onera project aiming at multidisciplinary team work on formation flying radar antennas, the initial mission chosen has been GMTI (Ground Motion Target Indicator) provided by STAP algorithm (Space Time Adaptive Processing) [1].

We will consider that the information available to each vehicle is restricted to its neighbour: with the leader-follower approach that we have taken from the beginning, that means that each satellite measures the relative position of the preceding one and acts through the multivariable control law derived in the preceding section, on the basis of the ClohessyWiltshire model between the two vehicles involved.

It must be clear that this situation, which uses an optical or radio-frequency relative metrology, is worse than the case of relative information between each satellite and the leader, as would provide e.g. the availability of a differential Global Positioning System. In the latter case, there is no dynamic coupling between the loops due to relative motion. Ensuring the cohesion of the formation with local measurements is

ONERA, System Control and Flight Dynamics Department, BP 4025, F31055 Toulouse Cedex, France fabrice. demouranteonera. fr and jean-pierre.chretien@onera.fr a problem seen in the more general case of land or aerial vehicle platoons.

This falls in the framework of structured synthesis: from input-output point of view the control law is block diagonal, and a technique able to build up a block-diagonal control law more efficient that the plain independent set of local control laws is needed. In other words the objective is to obtain a controller for each satellite, then a local control, in order to satisfy a global performance criterion on the satellites formation.

The literature about structured control laws is abundant and there are as many methodologies as papers. Many of these techniques are LMI based and they are not necessarly easy to implement, especially when there are different kinds of specifications and/or high order models. Besides these approaches are valid when the control law has the same structure as the plant ([2], [12], [15], [11]), for static feedback [17], or specific structure of the plant and controller [16].

The other possibility is to adapt a classical methodology to the specific case of a structured control law as for instance in [18], [10]. Nevertheless this kind of approach leads to high order control law with the difficulty to satisfy performance and robustness specifications on the overall plant.

In several papers specific technics for satellites formation have been developped like for instance an adaptative control to insure stability in a parameter varying contexte [13], [20], an LQ synthesis limited to 2 satellites formation [14], [19] with an extension in [4].

In this paper an innovative methodology is involved to control a along track satellite formations in a circular low earth orbit. The main problem is to insure a global performance with local controllers in spite of non measured perturbation as air drag.

So the Youla or $Q$ parametrization is considered. Convex synthesis [3], [6], a Youla-parameter based technique, is similar to the $H_{\infty}$ synthesis, since convex synthesis allows to weight closed loop transfer matrices. Thanks to this approach it is possible to extend the previous methodology to the case with four satellites in the same line as the weighted $H_{\infty}$ synthesis. Even if, in the general case, to obtain a decentralized dynamic output feedback without constraint on the plant, the problem is not convex in $Q$, it is possible thanks a specific choice on $Q$ to make convex in $Q$ the problem with a slight conservatism. Besides it is possible also to take into account a priori and explicitly time and frequency domain constraints and then to satisify complex specifications in a structured context much more easier than an LQ approach. Let us notice that formation control is 
obtained without velocity measurements to reduce the cost and mass needed.

The paper is organized as follows: in the first section, we will briefly recall the well known results on relative formation flying, and set the landscape for control design by giving an insight on disturbance relative importance.

The second section is devoted to control design. The first problem is to synthesize a control law for a pair of satellites an $H_{\infty}$ synthesis has been used to take into account several kinds of specifications as closed loop damping and disturbance rejection with a MIMO model.

The third section is devoted to the extension of the methodology to more than two satellites, in a situation where each satellite knows only its neighbour.

\section{ORBITAL MOTION AND FORMATION FLYING}

\section{A. Equations of motion}

The equations describing the relative motion of one spacecraft w.r.t. another one in a local frame (radial, along-track, across-track) have been extensively used for years in the context of RDV \& Docking studies.

1) Choice of parameters: Let us consider the relative motion of a follower satellite $M_{2}$ of mass $m_{2}$ w.r.t. a leader satellite $M_{1}$ with $x, y$ and $z$ which respectively represent the radial, the aong track and the cross-track distance. The local frame is built from the local vertical and the orbit normal vector.

2) Clohessy-Wiltshire equations: Indeed, if the orbit is circular,

- the true anomaly rate $\dot{\nu}$ is a constant and $\ddot{\nu}=0$,

- the gravity potential is spherical.

We use the more usual expression relating true displacements to accelerations:

$$
\left\{\begin{aligned}
& \ddot{x}-2 n \dot{y}-3 n^{2} x \simeq \gamma_{x} \\
& \ddot{y}+2 n \dot{x}+\gamma_{y} \\
& \ddot{z}+n^{2} z
\end{aligned}\right.
$$

As the system is linear, the transfer function can be computed and reads

$$
\frac{\mathbf{r}}{\mathbf{u}}=\left[\begin{array}{ccc}
\frac{n^{2}}{s^{2}+n^{2}} & 2 \frac{n^{3}}{s\left(s^{2}+n^{2}\right)} & 0 \\
-2 \frac{n^{3}}{s\left(s^{2}+n^{2}\right)} & -\frac{\left(3 n^{2}-s^{2}\right) n^{2}}{\left(s^{2}+n^{2}\right) s^{2}} & 0 \\
0 & 0 & \frac{n^{2}}{s^{2}+n^{2}}
\end{array}\right]
$$

which enforces the result, already visible on the set of differential equations (1), that the across-track relative motion is decoupled from the in-plane relative motion. Equation (2) shows the modes with twice a pair of complex conjugate modes at the orbital frequency $n$ and one double integrator. So the system in conservative (all modes imaginary), but unstable because of the double integrator - the multiple modes at the orbital frequency belong to separate Jordan blocks in the state space canonical decomposition because they correspond to independent motions on orthogonal axes.

So here is the system for which we would like to find a control design: a 4 state, two-input, two-output system with high coupling at low frequency between altitude error and along-track error.

The true multivariable nature of the problem and the limits of the axis by axis control design grounded on phase plane techniques has been discussed in [5], together with a preliminary discussion on modal or $H_{2}$ techniques applicability to the multivariable problem. In the next section a solution using the $H_{\infty}$ framework is proposed.

\section{IN-PLANE CONTROL DESIGN FOR TWO SATELLITES}

\section{A. Control design issues}

The system dynamics described by the Clohessy-Wiltshire equations are linear. The control problem is mostly a disturbance rejection problem, as we have seen in the preceding section that the atmospheric drag is seen as non measured low frequency disturbance at the system input. The gravity gradient is taken into account by the multivariable model.

The control problem for the case of two satellites has been widely developed in [8] whereas only the main characteristics of this synthesis are presented in this section.

1) Control synthesis model: The 2 inputs are accelerations in $x$ and $y$. The outputs are the positions $x$ and $y$ of the follower satellite. Recall that $x$ and $y$ respectively represent the radial distance and along-track distance. The linear model is of order 4.

2) Specifications: Let us recall first that the linear model represents the relative motion of the follower satellite w.r.t. the leader satellite. In the ROMULUS context the along track distance between 2 satellites is $10 \mathrm{~m}$. The maximal error is 1 $m$. The disturbance must be rejected within an orbital period, i.e. $6000 s$.

\section{B. $H_{\infty}$ Synthesis}

1) Methodology: The specifications are represented in the $H_{\infty}$ synthesis scheme by the following characteristics:

- Transfer $T_{p \rightarrow y}$ allows to satisfy the specification on the disturbance rejection. This transfer corresponds to $G S_{u}$ where $G$ is the model to control and $S_{u}=T_{p \rightarrow u}$ is the input sensitivity function $(I+K G)^{-1}$.

- Transfer matrix $T_{n \rightarrow u}$ allows to control the command effort for noise on output $y$.

Let us notice that we have only a specification on the along-track axis, then $W_{0}(s)$ is a $2 \times 2$ function matrix of order 1 and $W_{3}(s)$ is a $2 \times 2$ function matrix of order 2 since we want to act on actuators in $x$ and $y$. Others weighting functions are only constant gains. The control law is of order $4+3=7$. After a short reduction step it is possible to have a 5 order controller.

2) Results: Closed loop poles are given by table I. Time domain simulations are given by Fig. 1 .

We notice that these results satisfy specifications. In brief the control law ensures disturbance rejection, a sufficient closed loop bandwidth, and a very satisfactory closed loop poles damping. 


\begin{tabular}{ccc}
\hline Eigenvalue & Damping & Frequency $(\mathrm{rad} / \mathrm{s})$ \\
\hline$-2.24 \mathrm{e}-03$ & 1.00 & $2.24 \mathrm{e}-03$ \\
$-2.07 \mathrm{e}-03+2.43 \mathrm{e}-03 \mathrm{i}$ & 0.65 & $3.19 \mathrm{e}-3$ \\
$-2.07 \mathrm{e}-03-2.43 \mathrm{e}-03 \mathrm{i}$ & 0.65 & $3.19 \mathrm{e}-3$ \\
$-4.86 \mathrm{e}-03$ & 1.00 & $4.86 \mathrm{e}-03$ \\
$-6.36 \mathrm{e}-03$ & 1.00 & $6.36 \mathrm{e}-03$ \\
$-2.00 \mathrm{e}-02$ & 1.00 & $2.00 \mathrm{e}-02$ \\
$-2.00 \mathrm{e}-02$ & 1.00 & $2.00 \mathrm{e}-02$ \\
$-6.18 \mathrm{e}-02$ & 1.00 & $6.18 \mathrm{e}-02$ \\
$-6.18 \mathrm{e}-02$ & 1.00 & $6.18 \mathrm{e}-02$ \\
\hline
\end{tabular}

TABLE I

CLOSED LOOP POLES

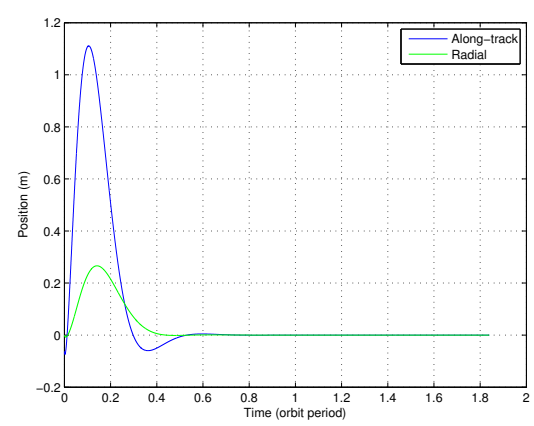

Fig. 1. Relative motion

\section{STRUCTURED SYNTHESIS BY YOULA-PARAMETRIZATION}

\section{A. Introduction}

We have set up a control synthesis methodology which gives satisfactory results in the case of a formation of two satellites. Now we will face the more complicated problem of the 4-satellites Romulus formation.

Weighting functions, closed loop analysis and performance/robustness trade-off are similar to those of $H_{\infty}$ synthesis. The advantages of this approach for our problem are numerous:

- it is possible to take into account different kind of specifications ( $H_{\infty}, l_{\infty}, H_{2}$ etc.) very easily;

- this approach is very close to the classical weighted $H_{\infty}$ synthesis, thus it is possible to use the methodological background developed for 2 satellites in the preceding section for the 4 satellites formation control;

- it is prone to structured control synthesis thanks to the Youla-parameter.

\section{B. Principle}

Let us consider a classical standard form where $y(t)$ and $u(t)$ are the inputs/outputs of the control law and $w(t)$ and $z(t)$ are the closed loop inputs/outputs to control. $P(s)$ represents the synthesis model with weighting functions chosen from robustness and performance specifications and $K_{0}$ represents an available control law. Two hypothesises are necessary to allow the use of convex synthesis methodology:

- the transfer matrix $P(s)$ should be proper;
- the initial controller $K_{0}$ should ensure closed loop stability.

Let us split transfer matrix $P$ in the following way:

$$
P=\left[\begin{array}{ll}
P_{11} & P_{12} \\
P_{21} & P_{22}
\end{array}\right]
$$

It is possible to write the transfer matrix between $w$ and $z$ as a function of $P$ and any controller $K$ by the lower linear fractional transformation $F_{l}(P, K)=T_{w \rightarrow z}=$ $P_{11}+P_{12} K\left(I-P_{22} K\right)^{-1} P_{21}$. The problem is to determine the control law $K$ which satisfies specifications on $T_{w \rightarrow z}$, which is deeply non linear in $K$. We will now show that Qparametrization allows to express the closed loop constraints as a linear expression in $Q$ :

$$
F_{l}(P, K)=T_{1}-T_{2} Q T_{3}
$$

where $Q$ becomes the synthesis parameter and $T_{1}, T_{2}$ an $T_{3}$ contain the poles of the initial closed loop system. In fact Q-parametrization allows to substitute $Q$ to $K$ to make the optimisation problem convex.

\section{Time and frequency domain responses affinity.}

We have shown that closed loop transfer matrix is affine in $Q$ (4). $Q$ can be parametrized as $Q=\sum_{i=1}^{n} \theta_{i} Q_{i}$, where $Q_{i}$ are filters whose poles are determined a priori and $\theta_{i}$ are optimization parameters. The set of these filters is a base which is used to build $Q$.

- Time domain constraints: for instance, consider the time domain closed loop response, we can write $z(t)=$ $z^{0}(t)+\sum_{i=1}^{n} \theta_{i} z^{i}(t)$ where $z^{0}(t)=T_{1} w(t)$ and $z^{i}(t)=$ $T_{2} Q_{i} T_{3} w(t)$.

- Frequency domain constraints: let us consider the general case of optimization/minimization of $H_{\infty}$ norm, i.e. the minimization of $\gamma$ under the constraint:

$$
\bar{\sigma}[T(j \omega)] \leq \gamma \alpha(j \omega) \quad \forall \omega \in\left[\omega_{1}, \omega_{2}\right]
$$

To solve this problem the cutting planes method [3], [9] is used. The idea is to approximate the non differentiable convex constraint at $\theta=\theta^{0}$ by an affine one, $\bar{\sigma}\left[T\left(j \omega, \theta^{0}\right)\right]+S^{T}\left(\theta-\theta^{0}\right) \leq \gamma \alpha(\omega)$, where $S$ is called the sub-gradient.

When approximating (5) at different points $\theta=\theta^{i}$ and different frequencies $\omega=\omega_{i}$ all these affine constraints can be stacked into an LP problem $A\left[\begin{array}{l}\theta \\ \gamma\end{array}\right] \leq b$.

\section{Choice of a base}

An orthonormal base is used, called Takenaka and Malmquist base, which combines properties of Laguerre and Kautz base. The decomposition of $Q_{i}(s)$ is given by (6).

$$
Q_{i}(s)=\frac{\sqrt{2 \Re\left(a_{i}\right)}}{s+a_{i}} \prod_{k=1}^{i-1} \frac{s-\overline{a_{k}}}{s+a_{k}}, \quad Q_{0}(s)=1, \quad a_{k} \in C^{+}
$$

where $a_{k}$ are the filters poles and are determined a priori to cover the frequency domain of the bandwidth. 


\section{E. Parametrization of stabilizing controllers}

Let us consider a double coprime factorization of $G$ which represents the system to control on $R H_{\infty}$, i.e. matrices $M_{l}$, $N_{l}, Y_{l}, M_{r}, N_{r}, X_{r}, Y_{r} \in R H_{\infty}$ such that $G=N_{r} M_{r}^{-1}=$ $M_{l}^{-1} N_{l}$ :

$$
\left[\begin{array}{cc}
X_{l} & -Y_{l} \\
-N_{l} & M_{l}
\end{array}\right]\left[\begin{array}{cc}
M_{r} & Y_{r} \\
N_{r} & X_{r}
\end{array}\right]=I
$$

The set of stabilizing control laws $K(Q)$ are parametrized by $Q$ in the following way:

$$
K(Q)=\left(Y_{r}-M_{r} Q\right)\left(X_{r}-N_{r} Q\right)^{-1}=\left(X_{l}-Q N_{l}\right)^{-1}\left(Y_{l}-Q M_{l}\right)
$$

where $\left(X_{l}-Q N_{l}\right)^{-1}$ and $\left(X_{r}-N_{r} Q\right)^{-1}$ are invertible and $Q \in R H_{\infty}$.

Now we must express the transfer matrices $T_{1}, T_{2}, T_{3}$ as functions of the coprime factors. If the closed loop transfer matrices are given by (4), then $T_{1}, T_{2}$ et $T_{3} \in R H_{\infty}$ are given by (9).

$$
T_{1}=P_{11}+P_{12} Y_{r} M_{l} P_{21} \quad T_{2}=P_{12} M_{r} \quad T_{3}=M_{l} P_{21}
$$

The coprime factorization is particularly easy to establish for a stable and stabilizing initial control law $K_{0}$. The following double coprime factorization can be used for $G$ and $K[15]$ :

$$
\begin{array}{cl}
M_{l}=\left(I-G K_{0}\right)^{-1} & M_{r}=-\left(I-K_{0} G\right)^{-1} \\
N_{l}=G\left(I-K_{0} G\right)^{-1} & N_{r}=-G\left(I-K_{0} G\right)^{-1} \\
X_{l}=-I \quad Y_{l}=-K_{0} & X_{r}=I \quad Y_{r}=K_{0}
\end{array}
$$

\section{F. Structure of $Q$ for decentralized controllers}

The question is: how to determine the structure of $Q$ which preserves the structure for $K(Q)$ ?

Let us suppose a block diagonal control law with 2 blocks. We have $G=M_{l}^{-1} N_{l}=N_{r} M_{r}^{-1}$ and $K_{0}=Y_{r} X_{r}^{-1}=$ $X_{l}^{-1} Y_{l}$. Equation (7) can be rewritten, in the decentralized case with:

$$
\begin{gathered}
M_{r}=\left[\begin{array}{l}
M_{r 1} \\
M_{r 2}
\end{array}\right]=\left[\begin{array}{ll}
M_{r 11} & M_{r 12} \\
M_{r 21} & M_{r 22}
\end{array}\right] \\
N_{r}=\left[\begin{array}{l}
N_{r 1} \\
N_{r 2}
\end{array}\right]=\left[\begin{array}{ll}
N_{r 11} & N_{r 12} \\
N_{r 21} & N_{r 22}
\end{array}\right]
\end{gathered}
$$

and $X_{r}=\operatorname{blockdiag}\left(X_{r 1}, X_{r 2}\right), \quad X_{l}=$ blockdiag $\left(X_{l 1}, X_{l 2}\right), \quad Y_{r}=\operatorname{blockdiag}\left(Y_{r 1}, Y_{r 2}\right)$ and $Y_{l}=\operatorname{blockdiag}\left(Y_{l 1}, Y_{l 2}\right) . M_{l}$ and $N_{l}$ are decomposed of the same way. If the left coprime decomposition of $G$ is performed the set of stabilizing decentralized control laws can be written with the left coprime decomposition $K(Q)=\left(X_{l}-Q Y_{l}\right)^{-1}\left(Y_{l}-Q M_{l}\right)$ where $\left(X_{l}-Q Y_{l}\right)$ is invertible and $Q \in Q_{l}$ such that [7]:

$$
\begin{aligned}
Q_{l} & =\left\{Q \mid Q=Q_{w}^{-1}\left[\begin{array}{cc}
Q_{1} & 0 \\
0 & Q_{2}
\end{array}\right]\right\} \\
Q_{w} & =\left[\begin{array}{cc}
Q_{11} & Q_{1} W_{12} \\
Q_{2} W_{21} & Q_{22}
\end{array}\right]
\end{aligned}
$$

where

$$
\begin{aligned}
& W_{12}=-N_{l 11} M_{r 12}+M_{l 11} N_{r 12}=l_{12} M_{r 22}-M_{l_{12}} N_{r 22} \\
& W_{21}=-N_{l 22} M_{r 21}+M_{l 22} N_{r 21}=N_{l 21} M_{r 11}-M_{l 21} N_{r 11}
\end{aligned}
$$

It is possible to obtain a similar relationship with a right decomposition. We notice that $Q$ is not necessarily block diagonal in spite of the block diagonal structure of $K(Q)$. The synthesis parameters of $Q$, i.e. $Q_{11}, Q_{22}, Q_{1}$ et $Q_{2}$ must satisfy non convex constraints. To restore convexity, it is possible to force constraints or values on a certain number of design parameters. Typically the constraints $Q_{2}=0, Q_{11}=$ $Q_{22}=I$ are imposed which make $Q$ block-diagonal

$$
Q=\left[\begin{array}{cc}
Q_{1} & 0 \\
0 & 0
\end{array}\right]
$$

It allows to tune channel 1 without modifying channel 2 , and it is possible to tune channel 2 after having modified channel 1. This approach, even if it suffers from certain conservatism, leads to various implementations [18], [10]. We are thus able to tune the different channels independently while taking into account their coupling.

\section{G. Romulus application}

1) System model and closed loop structure.: As the Clohessy-Wiltshire models are leader follower models we obtain for $G$ the following structure:

$$
G=\left[\begin{array}{ccc}
G_{11} & 0 & 0 \\
G_{21} & G_{22} & 0 \\
0 & G_{32} & G_{33}
\end{array}\right]
$$

where $G_{i j}$ is transfer matrix $2 \times 2$. The global control law is a 6 inputs/ 6 outputs structured control law with $2 \times 2$ blocks. The choice of the initial control law $K_{0}$ is the control law synthesized previously in section III-B.1, with $K_{0}=$ $\operatorname{diag}(K, K, K)$.

2) $H_{\infty}$ specifications:

1) Synthesis for the $3^{\text {rd }}$ satellite.

This problem can be described by the following specifications:

- To minimize: the closed loop transfer matrix between the drag input of the $2^{\text {nd }}$ and $3^{r d}$ satellites and the along-track displacement of the $3^{\text {rd }}$ satellite, that is minimize the $H_{\infty}$ norm of $G S_{u}(4,2)$ and $G S_{u}(4,4)$;

- To constrain: the closed loop transfer matrix between the drag input of the $2^{\text {nd }}$ and $3^{\text {rd }}$ satellites and the radial displacement of the $3^{\text {rd }}$ satellite, that is constrain the $H_{\infty}$ norm of $G S_{u}(3,2)$ and $G S_{u}(3,4)$.

We notice that two cost functions are minimized to desensitize the along-track state of $3^{r d}$ satellite and two constraints are added to control the radial state of the $3^{\text {rd }}$ satellite.

2) Synthesis for the $4^{\text {th }}$ satellite.

The procedure is similar to the previous one: 
- To minimize: $H_{\infty}$ norm of $G S_{u}(6,2), G S_{u}(6,4)$, and $G S_{u}(6,6)$;

- To constrain: $H_{\infty}$ norm of $G S_{u}(5,2), G S_{u}(5,4)$, and $G S_{u}(5,6)$.

To limit the $Q$ order only 2 complexe conjugate poles are chosen:

\begin{tabular}{ccc}
\hline Eigenvalue & Damping & Frequency $(\mathrm{rad} / \mathrm{s})$ \\
\hline$-1.13 \mathrm{e}-02 \pm 1.13 \mathrm{e}-02 \mathrm{i}$ & $7.07 \mathrm{e}-01$ & $1.60 \mathrm{e}-02$ \\
\hline & TABLE II \\
& POLES.
\end{tabular}

3) Results: To illustrate results the along-track relative position has been normalized to 1 .

We clearly notice, on Fig. 2 and 3, the significant improvement of the $3^{\text {rd }}$ satellite behavior for the along-track component since we are able reduce overshoot by $40 \%$.

- This improvement is done while preserving the block diagonal structure of the control law

$$
K_{d}(Q)=\left[\begin{array}{ccc}
K & 0 & 0 \\
0 & K_{1}(Q) & 0 \\
0 & 0 & K
\end{array}\right]
$$

The $K(Q)$ synthesis has been performed without modification of $K$ while taking into account the interconnection of $G$ with $K$.

- $K_{1}(Q)$ order is 8 what is a limited order.

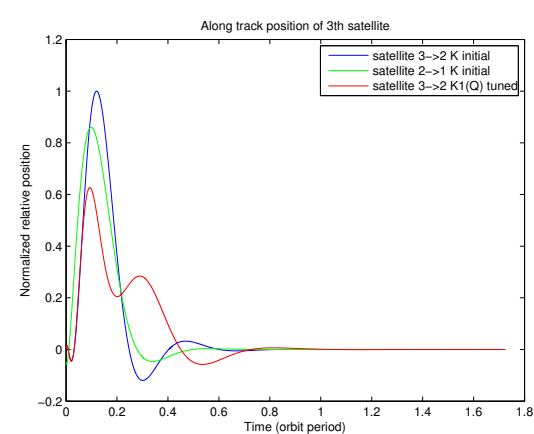

Fig. 2. $3^{\text {rd }}$ satellite along-track relative position

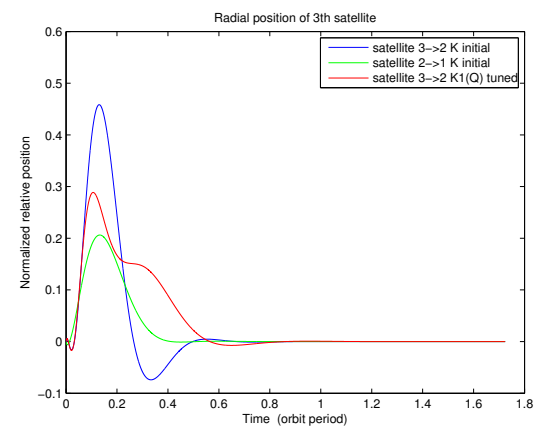

Fig. 3. $3^{\text {rd }}$ satellite radial relative position

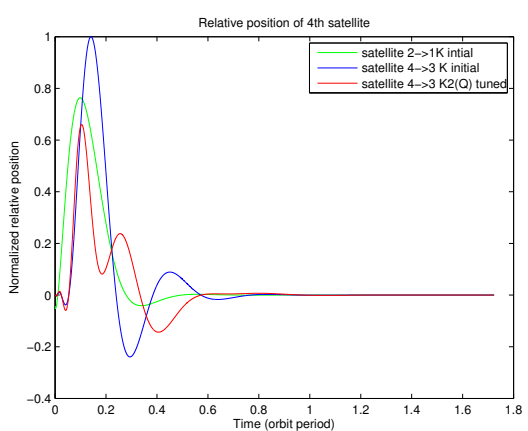

Fig. 4. $4^{r d}$ satellite along-track relative position

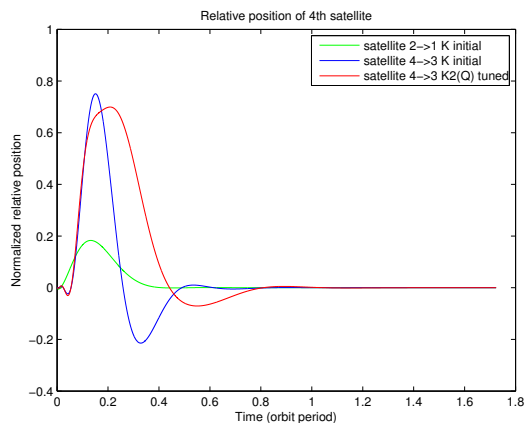

Fig. 5. $4^{r d}$ satellite radial relative position

Now the $4^{\text {th }}$ satellite control law is tuned.

Results are shown on Fig. 4 and 5. We can notice an improvement of $40 \%$ of the $4^{t h}$ satellite along-track component. The structure of the control law is still block diagonal

$$
K_{d}(Q)=\left[\begin{array}{ccc}
K & 0 & 0 \\
0 & K_{1}(Q) & 0 \\
0 & 0 & K_{2}(Q)
\end{array}\right]
$$

$K_{1}(Q)$ and $K$ are not modified by the $K_{2}(Q)$ synthesis as in an independent synthesis. The $K_{2}(Q)$ order is the same as the $K_{1}(Q)$ order. However the $K_{2}(Q)$ synthesis has been performed while taking into account the interconnection of $G$ with $K$ and $K_{1}(Q)$. Recall that $K$ is of order 5 and $K_{1}(Q)$ and $K_{2}(Q)$ are of order 8 . In other words we are able to improve performance of the structured closed loop significantly with a limited contol law order.

At last a mixed synthesis $H_{\infty} / l_{\infty}$ has been done. Outputs are constrained to comply to a time domain template: we can see on Fig. 6 and 7 that the time domain constraint on the along-track and radial state are perfectly respected. In this synthesis, the $H_{\infty}$ minimization of $G S_{u}(4,4)$ and the $H_{\infty}$ constraint of $G S_{u}(3,4)$ have been replaced by $l_{\infty}$ constraints. We can notice that it is easier to minimize/constrain the along-track displacement of the $3^{r d}$ satellite than in the previous case. Nevertheless it is more difficult to control the radial displacement (Fig. 7) whereas the along track displacement presents oscillating behavior. In brief the choice of $l_{\infty}$ or $H_{\infty}$ specifications depend on specifications. If to 


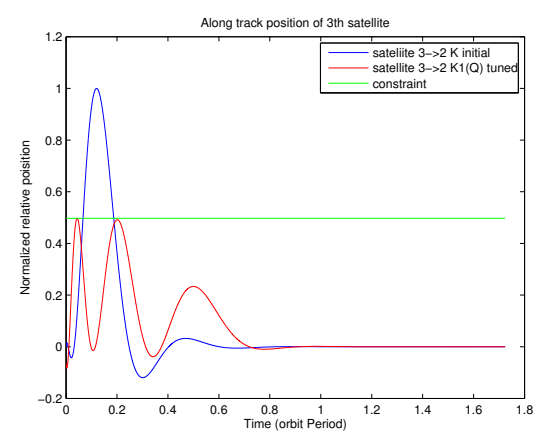

Fig. 6. $3^{\text {rd }}$ satellite along-track relative position

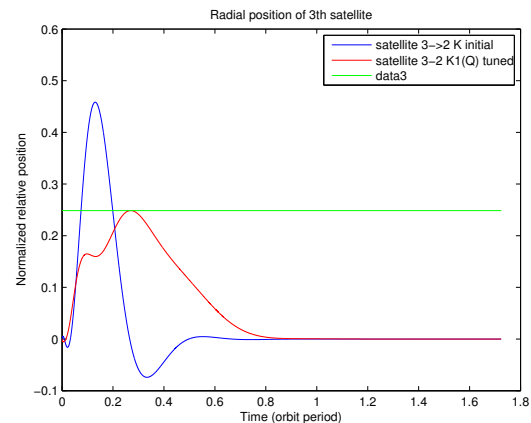

Fig. 7. $3^{\text {rd }}$ satellite radial relative position

constraint $l_{\infty}$ norm of specific closed loop transfer functions is the most important specification it can be interesting to use $l_{\infty}$ constraints in the optimization problem.

\section{CONCLUSION}

A specific approach has been developed to synthesize a control law for a multi-satellite formation, in the low altitude along track configuration.

The Clohessy-Wiltshire model, describing the relative motion of a follower satellite w.r.t. a leader satellite is used for control design and validation. An $H_{\infty}$ technique is involved to treat the case of 2 satellites.

But it is important to treat the problem with more that two satellites: the control of a platoon of satellites turns to structured control (decentralized control of four satellites in our case) when each satellite is the follower of the preceding one and has no global positioning measurements. We used a convex synthesis technique based on the Youla-parameter $Q$ to tackle this problem. Thanks to a specific structure for $Q$, a block diagonal control law is optimized. Each diagonal control law is first obtained using the controller obtained in the two-satellites case. Then convex optimization of the $Q$ parameter of each channel allows to improve the formation performance in a significant way with a limited control law order. This technic allows to combine the advantages of the sequential and independant synthesis witout their drawbacks. Besides from methodological point of view the Youla partameter synthesis is very similar to classical technics like
$H_{\infty} / H_{2}$ synthesis. In other words it is not necessary to develop another methodology to involve the structured $Q$ synthesis, it is just enough to resume and adapt it to the structured case.

Lastly we have shown that it was possible to deal efficiently with time domain constraints, which may be used to obtain a threshold behaviour similar to what is usually obtained in the case of mono-axis non-linear phase plane control.

\section{REFERENCES}

[1] J.-P. Aguttes, S. Attia, F. Douchin, B. Vaizan, J.-D. Desjonquères, J.-P. Chrétien, T. Amiot, and C. Tison. Romulus : Along track formation of radar satellites for mti (moving target identification) and high sar performance. In Intrernational Geosciences And Remote Sensing Symposium, Denver, Co, USA, 2006. IEEE.

[2] B. Bamiech, F. Paganini, and M.A. Dahleh. Distributed control of spacially invariant systems. IEEE Transactions on Automatic Control, 47(7):1091-1107, 2002.

[3] S. Boyd and C. Barrat. Linear controller design: limits of performance. Prentice Hall, 1990.

[4] J.R. Carpenter. Decentralized control of satellite formations. International Journal of Robust and Nonlinear Control, 12(2):141-161, 2002.

[5] J.-P. Chrétien. Closed loop relative motion control for a segmented along-track radar antenna in low earth orbit. In 6th International ESA Conference on Guidance, Navigation and Control Systems, Loutraki, Greece, 17 - 20 October 2005. ESA.

[6] I. Dardenne and G. Ferreres. Design of a lateral flight control system for a highly flexible aircraft using convex synthesis. Proc. of the 1998 ICAS (International Council of the Aeronautical Sciences), 1998.

[7] A. Date and J.H. Chow. Decentralized stable factors and a paramterization of decentralized controllers. IEEE Transactions on Automatic Control, 39(2):347-351, 1994.

[8] F. Demourant and J-P. Chrétien. Relative motion control for alongtrack formation flight in low earth orbit: Linear synthesis with nonlinear actuators. 3rd International Symposium on Formation Flying, Missions and Technologies, European Space Agency conference, 23-25 April, Noordwijk ESA-ESTEC, 2008.

[9] G. Ferreres and G. Puyou. Flight control law design for a flexible aircraft: limits of performance. Journal of Guidance, Control and Dynamics, 29(4):870-878, 2006.

[10] M. Hovd and S. Skogestad. Sequential design of decentralized controllers. Automatica, 30(10):1601-1607, 1994.

[11] C. Langbort, R.S. Chandra, and R. D'Andrea. Distributed control design for systems interconnected over an arbitrary graph. IEEE Transactions on Automatic Control, 49(9):1502-1518, 2004.

[12] X. Qi, M.V. Salapaka, P.G. Voulgaris, and M. Khammash. Structured optimal and robust control with multiple criteria: a convex solution. IEEE Transactions on Automatic Control, 49(10):1623-1639, 2004.

[13] M.S. Queiroz, V. Kapila, and Q. Yan. Adaptative nonlinear control of multiple spacecraft formation flying. Journal of Guidance, Control and Dynamics, 23(3):385-389, 2000.

[14] D.C. Redding, N.J. Adams, and E.T. Kubiak. Linear-quadratic stationkeeping for sts orbiter. Journal of Guidance, Control and Dynamics, 12(2):248-256, 1989.

[15] M. Rotkowitz and S. Lall. A characterisation of convex problems in decentralized control. IEEE Transactions on Automatic Control, 50(12):1984-1996, 2005.

[16] C.W. Scherer. Design of structured controllers with applications Proceedings of the IEEE, pages 5204-5209, December 2000.

[17] G. Scorletti and G. Duc. An lmi approach to decentralized $h_{\infty}$ control. International Journal of Control, 74(3):211-224, 2001.

[18] S. Skogestad and M. Morari. Robust performance of decentralized control system by independent design. Automatica, 25(1):119-125, 1989.

[19] R.H. Vassar and R.B. Sherwood. Formationkeeping for a pair of satellites in a circular orbit. Journal of Guidance, Control and Dynamics, 8(2):235-242, 1985

[20] H. Wong, V. Kapila, and A.G. Sparks. Adaptative output feedback tracking control of spacecraft formation. International Journal of Robust and Nonlinear Control, 12(2):117-139, 2002. 